\title{
Positive effect of curcumin on inflammation and mitochondrial dysfunction in obese mice with liver steatosis
}

\author{
JONG-JEN KUO ${ }^{1,2}$, HEN-HONG CHANG ${ }^{1-3}$, TUNG-HU TSAI ${ }^{1}$ and TZUNG-YAN LEE ${ }^{3}$ \\ ${ }^{1}$ Institute of Traditional Medicine, National Yang Ming University, Taipei; ${ }^{2}$ Center for Traditional Chinese Medicine, \\ Chang Gung Memorial Hospital; ${ }^{3}$ Graduate Institute of Traditional Chinese Medicine, \\ Chang Gung University, Taoyuan, Taiwan, R.O.C.
}

Received April 4, 2012; Accepted June 8, 2012

DOI: $10.3892 / \mathrm{ijmm} .2012 .1049$

\begin{abstract}
Obesity contributes to the increased risk of liverrelated morbidity and mortality. The accumulation of macrophages in adipose tissue in an inflammatory state is a hallmark of obesity-induced hepatic steatosis. In this study, we reveal the role of curcumin in the molecular mechanisms underlying inflammatory events in a model consisting of obese mice with hepatic steatosis. Obese mice fed with curcumin experienced significant weight loss and significantly reduced serum triglyceride (TG) levels. The adipose tumor necrocis factor- $\alpha$, interleukin-6 (IL-6) and monocyte chemotactic protein-1 levels were significantly higher in obese mice compared to mice fed with curcumin. Compared to the obese mice, curcumin decreased the number of F4/80-positive macrophages in epididymal adipose and liver tissue. The induction of signal transducer and activator of transcription 3 phosphorylation by curcumin resulted in the downregulation of the suppressor of cytokine signaling 3 in the liver of obese mice. Curcumin decreased hepatic TG in obese mice by downregulating the gene expression of sterol regulatory element-binding protein-1c in the liver. The hepatic expression of several mitochondrial biogenesis genes decreased in the obese mice, including mitochondrial DNA (mtDNA), nuclear respiratory factor 1 (NRF1) and mitochondrial transcription factor A (Tfam), which are responsible for the lower mitochondrial respiratory chain (MRC) complex I activity and adenosine triphosphate production. By contrast, obese mice treated with curcumin showed normalized mtDNA, NRF1 and Tfam gene expression, reduced hepatic nuclear factor $-\kappa \mathrm{B}$ activities and levels of thiobarbituric acid reactive substances (TBARS) and restored mitochondrial oxidative metabolism and biogenesis. The results from the present sudy show that curcumin prevents fatty liver disease through multiple mechanisms, and suggest that curcumin may
\end{abstract}

Correspondence to: Professor Tzung-Yan Lee, Graduate Institute of Traditional Chinese Medicine, Chang Gung University, 259 Wen-Hwa 1st Road, Kweishan, Taoyuan 333, Taiwan, R.O.C.

E-mail: joyamen@mail.cgu.edu.tw

Key words: inflammation, mitochondrial dysfunction, curcumin, obesity, liver steatosis be used to prevent the development and progression of nonalcoholic fatty liver disease (NAFLD).

\section{Introduction}

The prevalence of non-alcoholic fatty liver disease (NAFLD) is considered one of the manifestations of metabolic syndrome. It is currently recognized that NAFLD is a common metabolic change that increases the risk of chronic liver diseases resulting from excessive fat accumulation in the liver. A number of studies have been published linking adiposity with inflammation and increased liver disease. Visceral white adipose tissue (AT) is currently considered the key depot linked with obesity-related systemic metabolic disturbances. Obesity is associated with chronic inflammation, evidenced by increased levels of chemokines/cytokines in AT and liver and increased accumulation and activation of macrophages in AT and liver (1-3). NAFLD is closely linked to obesity, diabetes, hyperlipidemia, insulin resistance and is considered to represent a hepatic manifestation of metabolic syndrome (4). In addition, excess fat accumulation in hepatocytes may lead to hepatocellular injury via direct cellular cytotoxicity mediated by free fatty acids (FFAs), oxidative stress, lipid peroxidation, mitochondrial impairment and cytokine-induced hepatotoxicity $(5,6)$. Furthermore, excessive fat accumulation in the liver damages mitochondria, which are the primary cellular sites for fatty acid utilization (7). Mitochondrial dysfunction may also play a crucial role in the induction of a 'two-hit' model, as mitochondria are involved in the $\beta$-oxidation of FFAs and are the most important cellular source of reactive oxygen species (ROS). In this study, we aimed to find treatments that would improve mitochondrial function in fatty liver disease in obese mice.

Recently, a role of the inflammatory response in obesity and non-alcoholic steatohepatitis (NASH) was established. Currently, inflammation overactivity in both the liver and AT is considered to contribute to metabolic disorders. The pharmacological blockade of the inflammation has shed light on the treatment of obesity and NASH $(8,9)$ by acting on the adipose and liver tissue. The polyphenol, curcumin, is generally regarded as having potent antioxidant, anti-inflammatory, and anti-carcinogenic properties (10). Curcumin modulates multiple molecular targets and has potent anti-inflammatory 
activities, which may contribute to its therapeutic role in obesity and obesity-related metabolic diseases. Despite the potential importance of curcumin in regulating inflammatory signaling and lipid metabolism, its role in controlling obesity-related hepatic inflammation has not been examined in vivo. Previous studies have shown that curcumin reduces the expression of certain inflammatory molecules in obese animals $(11,12)$. However, these studies did not establish the curcumin effect associated with lipotoxicity-induced mitochondrial abnormalities that sensitize the liver to additional pro-inflammatory insults. Therefore, we conducted the current study to examine the hypothesis that increased macrophage infiltration in the liver of obese mice induces inflammatory response and impairs mitochondrial function in the liver tissue. These results demonstrate that curcumin is a critical regulator of obesity-induced inflammation and mitochondrial biogenesis in obese mice.

\section{Materials and methods}

Experimental animals. C57BL/6J ob/ob mice have a mutation that prevents leptin synthesis, resulting in a phenotype of obesity, hepatomegaly, severe steatosis and high serum levels of aminotransferase (13). Male ob/ob C57BL/6J mice were obtained from the Jackson Laboratory (Bar Harbor, ME). Mice were housed 5/cage and maintained under a 12-h light, 12-h dark cycle with ad libitum access to food and water. Upon receipt at the age of 8 weeks, the mice were randomized to receive a normal diet. At the age of 13 weeks, these mice were further randomized as regards an addition to their predesignated diet of a 1 or $3 \%$ by weight admixture of curcumin or no additive. Curcumin was purchased from Sigma (St. Louis, MO), and the purity was $>94 \%$. Curcumin was dissolved in corn-oil (final concentration of corn-oil $<1 \%$ ) and mixed with the homogenized normal diet to form the final concentration of $3 \%(\mathrm{w} / \mathrm{w})$. This concentration showed the most protective effect on obesity-associated inflammation as previously reported (12). The animals were divided into 4 groups: i) wild-type mice fed with a normal diet, ii) $o b / o b$ mice fed with a normal diet, iii) ob/ob mice fed with a curcumin-supplemented diet (1\%) and iv) ob/ob mice fed with a curcumin-supplemented diet (3\%). Each experimental group consisted of 5 animals and all mice remained on their assigned diet until they were sacrificed by $\mathrm{CO}_{2}$ asphyxiation at the age of 21 weeks. All protocols were conducted in accordance with the Guide for the Care and Use of Laboratory Animals and were approved by the Chang Gung University Animal Care and Use Committee.

Biochemical measurement, histological and immunohistochemical analysis. Epididymal fat pads and liver tissue were harvested immediately after euthanization, weighed, flash frozen in liquid nitrogen, and stored at $-80^{\circ} \mathrm{C}$. Liver tissue was fixed in $10 \%$ formalin, embedded in paraffin, cut into 5- $\mu \mathrm{m}$-thick sections, and stained with hematoxylin and eosin (H\&E). Staining was performed using standard techniques by the Pathology Core of the Research Center at the Chang Gung Memorial Hospital and examined under a light microscopy by an experienced pathologist. Immunostaining for F4/80 (BioLegend, San Diego, CA) and neutrophil (Abcam,
Cambridge, MA) was performed in sections using the specific antibodies and an avidin-biotin complex immunoperoxidase method. Changes in glutathione (GSH) levels were determined by high-performance liquid chromatography (HPLC) (14). The thiobarbituric acid reactive substances (TBARS) were determined as described previously (15). To determine the serum alanine aminotransferase (ALT) levels, commercial kits (Roche Diagnostics GmbH, Manheim, Germany) were used. The concentrations of adipose tumor necrocis factor- $\alpha$ (TNF- $\alpha$ ), monocyte chemotactic protein-1 (MCP-1), and interleukin-6 (IL-6) were determined using sandwich ELISA. The capture and detection antibodies against rat TNF- $\alpha$, MCP-1 and IL-6 were purchased from $\mathrm{R} \& \mathrm{D}$ systems (Minneapolis, $\mathrm{MN}$ ).

Oil Red $O$ staining and liver triglyceride (TG) content. To visualize the fat droplet accumulation, frozen liver tissue sections were stained with Oil Red O. Liver samples were used to assay TG concentration using the Triglyceride Quantification kit (Randox, Antrim, UK).

$N F-\kappa B$ activity assay. Nuclear protein extracts were prepared from lean control liver tissue and curcumin-fed $o b / o b$ mice using the nuclear extract kit (BioVision, Mountain View, CA) according to the manufacturer's instructions. NF- $\kappa \mathrm{B}$ activity was determined by the NF- $\kappa$ B EZ-Transcription Factor assay (Millipore).

Reverse transcription-polymerase chain reaction ( $R T-P C R)$ and real-time $P C R$ analysis. Total RNA was extracted from the epididymal fat and hepatic tissue using the guanidinium-phenol-chloroform method. Total RNA $(5 \mu \mathrm{g})$ was reverse-transcribed using the RevertAid ${ }^{\mathrm{TM}}$ First Strand cDNA Synthesis kit according to the manufacturer's instructions. The cDNA was amplified using the TaqDNA polymerase kit (Fermentas, Vilnius, Lithuania). RT-PCR products were separated by electrophoresis on a $3 \%$ agarose gel and quantified by ImageQuant 5.2 software (Healthcare Bio-Sciences, Philadelphia, PA). Real-time PCR was performed on a LightCycler 1.5 apparatus (Roche Diagnostics $\mathrm{GmbH}$ ) using the LightCycler FastStart DNA MasterPLUS SYBR-Green I kit according to the manufacturer's protocol. Mitochondrial DNA (mtDNA) copy number was determined by real-time PCR as previously described (16).

Western blot analysis. Liver tissue was lysed with distilled water containing protease inhibitors and a Bio-Rad Rapid Coomassie kit was used to determine the total protein concentration. Protein $(50 \mu \mathrm{g})$ was run on a $10 \%$ SDS-polyacrylamide gel and transferred to a polyvinylidene difluoride membrane. Immunoblotting was performed with various mouse or rabbit monoclonal or polyclonal antibodies, followed by the incubation of the appropriate secondary antibody coupled with horseradish peroxidase. The blot was developed with a chemiluminescence system (ECL; Amersham, Piscataway, NJ) according to the manufacturer's instructions. The optical densities of the bands were quantified using the GS-700 Imaging Densitometer (Bio-Rad, Hercules, CA).

Mitochondrial respiratory complexes and adenosine triphosphate (ATP) measurement. The mitochondrial respiratory 

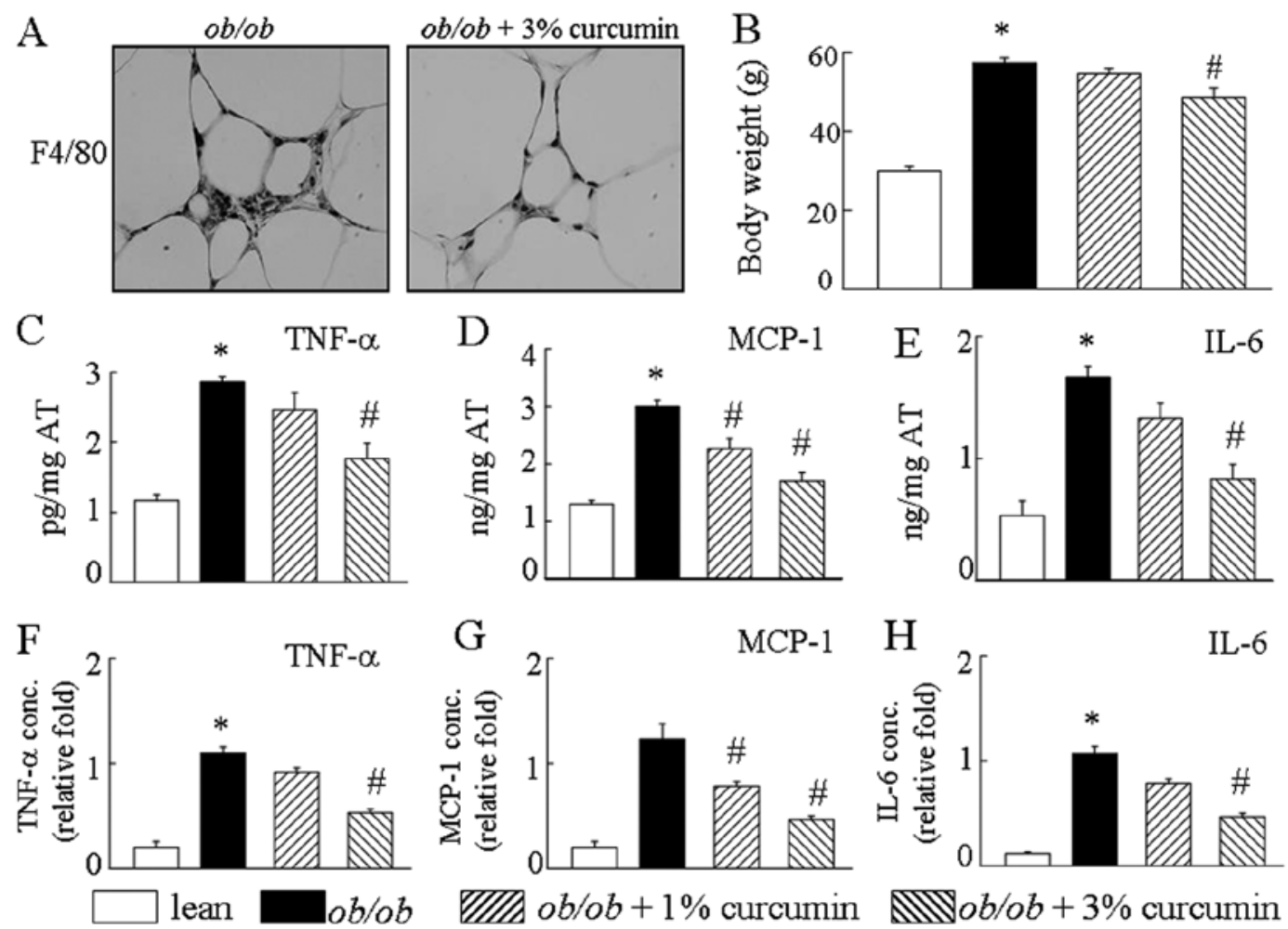

Figure 1. Effects of curcumin on body weight and cytokine gene expression of adipose tissue (AT) in obese mice. (A) AT of obese mice showed dark staining for F4/80, a macrophage marker, whereas the AT from mice treated with dietary curcumin showed significantly less macrophage staining. (B) Curcumin treatment was associated with significantly decreased body weights in the obese mice. TNF- $\alpha$, IL-6 and MCP-1 mRNA levels in AT of obese mice. (C-E) mRNA determined by real-time PCR in the obese or the curcumin-treated mice. Curcumin significantly reduced the secretion of cytokines in obese mice. (F) TNF- $\alpha$, (G) MCP-1 and (H) IL-6 concentrations were determined by specific ELISA. The results are expressed as the means \pm SEM of 2 different experiments performed in duplicate. " $\mathrm{P}<0.05$ compared with lean control mice and ${ }^{\prime \prime} \mathrm{P}<0.05$ compared with obese mice without curcumin treatment ( $\mathrm{n}=5$ ). TNF- $\alpha$, tumor necrocis factor- $\alpha$, MCP-1, monocyte chemotactic protein-1; IL-6, interleukin-6.

complex activity was analyzed as previously described (17). The ATP contents of liver homogenates were measured with the ATP bioluminescence assay kit HS II according to the manufacturer's instructions (Roche Diagnostics GmbH). The luminescence value was normalized by the protein concentration and the luminescence ratio was compared with the $o b / o b$ mice group.

Statistical analysis. Data were presented as the means \pm SEM. The statistical analysis were performed using a one-way analysis of variance followed by the Student Newman-Keuls multiple-range test. A value of $\mathrm{P}<0.05$ was considered to indicated a statistically significant difference.

\section{Results}

Consistent with previous reports (12), curcumin treatment of obese mice markedly induced weight loss as compared with the vehicle group (Fig. 1B). After an 8-week treatment with curcumin, the mice exhibited lower body weight, smaller epididymal fat pads, smaller livers and less total fat mass than obese mice. Compared to the lean mice, the $o b / o b$ mice had an increased number of F4/80-positive cells in the AT. Compared to the $o b / o b$ mice, the curcumin-treated groups had significantly different levels of macrophage cell markers (Fig. 1A). Additionally, obese mice han an increased production of MCP-1, IL- 6 and TNF- $\alpha$ by AT. Curcumin treatment in obese mice significantly decreased AT TNF- $\alpha$, IL-6, and MCP-1 mRNA levels compared to those in the curcumin-free controls (Fig. 1C-E). Treatment with curcumin caused a drastic reduction in the secretion of pro-inflammatory cytokines, including TNF- $\alpha$, MCP-1 and IL- 6 in the serum of obese mice, in a dose-dependent manner (Fig. 1F-H). Treatment with curcumin markedly decreased plasma and liver TG levels (Fig. 2C). Significant liver fatty degeneration and piecemeal necrosis of hepatocytes were observed in the obese mice (Fig. 2A). There were many red lipid deposits in the hepatocytes of the obese group. The number of lipid deposits decreased with curcumin, especially in the high-dose therapeutic groups (Fig. 2). Sterol regulatory element-binding protein-1c (SREBP-1c) mRNA levels tended to be higher in the liver tissue of obese mice than in lean mice (Fig. 2B). The expressions of the target genes of SREBP-1c, including fatty acid synthase (FAS) and acetyl-CoA carboxylase (ACC) were decreased in the liver of curcumintreated mice. Since the presence of low-grade inflammation is linked to the transcriptional activation of $\mathrm{NF}-\mathrm{\kappa B}$, we examined the effects of curcumin on NF- $\mathrm{kB}$ activity. Curcumin reduced NF- $\mathrm{KB}$ activity (Fig. 4C) in the liver tissue and decreased the production of the pro-inflammatory cytokine MCP-1, IL- 6 and TNF- $\alpha$ mRNA levels compared to obese mice (Fig. 3B). These data suggest that curcumin has a direct inhibitory effect on the chemokine expression in the liver. In addition, obese mice had higher levels of F4/80-positive cells, and a trend towards lower signal transducer and activator of transcription 3 (STAT3) 
A
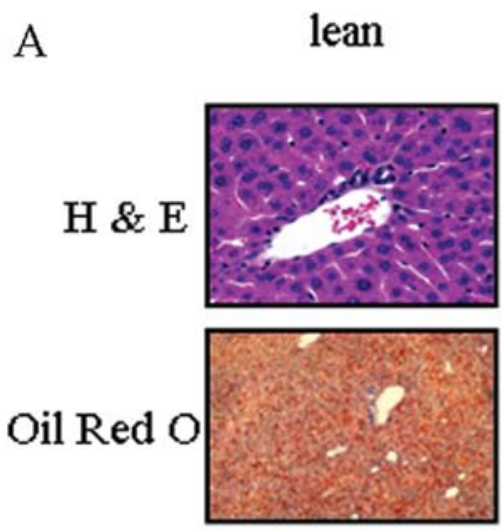

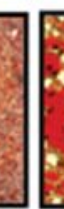
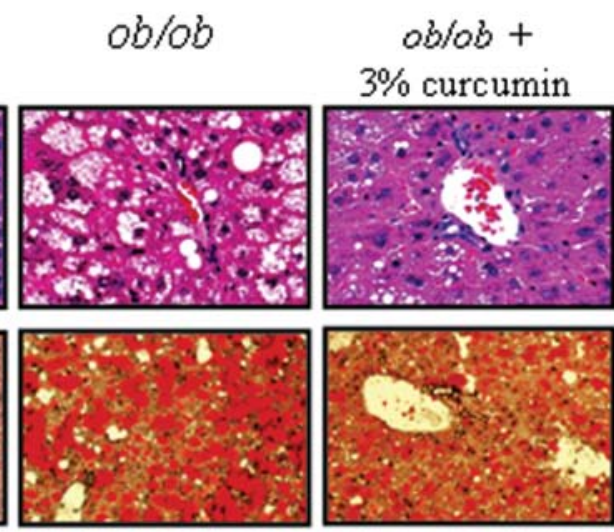

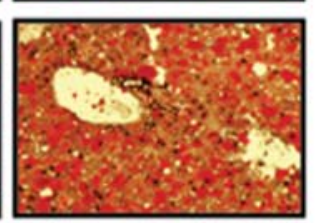

B

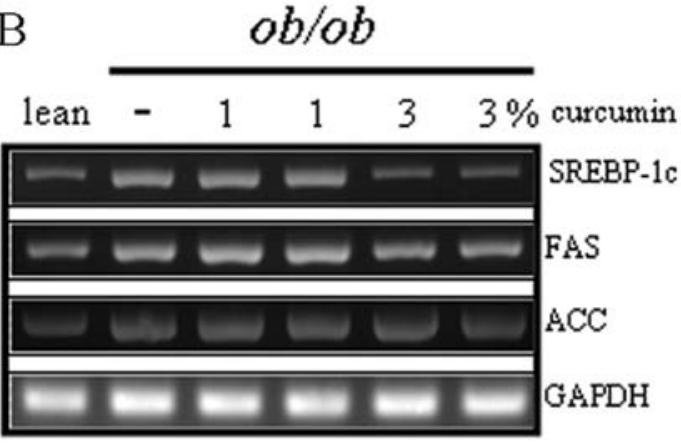

$\square$ lean $a b / a b$

Da $o b \% b+1 \%$ curcumin

$\Delta \circ b b+3 \%$ curcumin

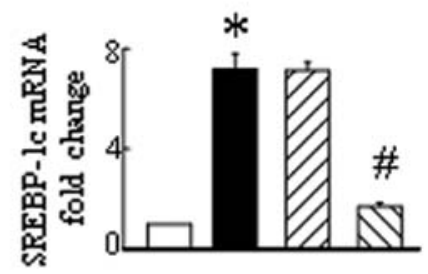

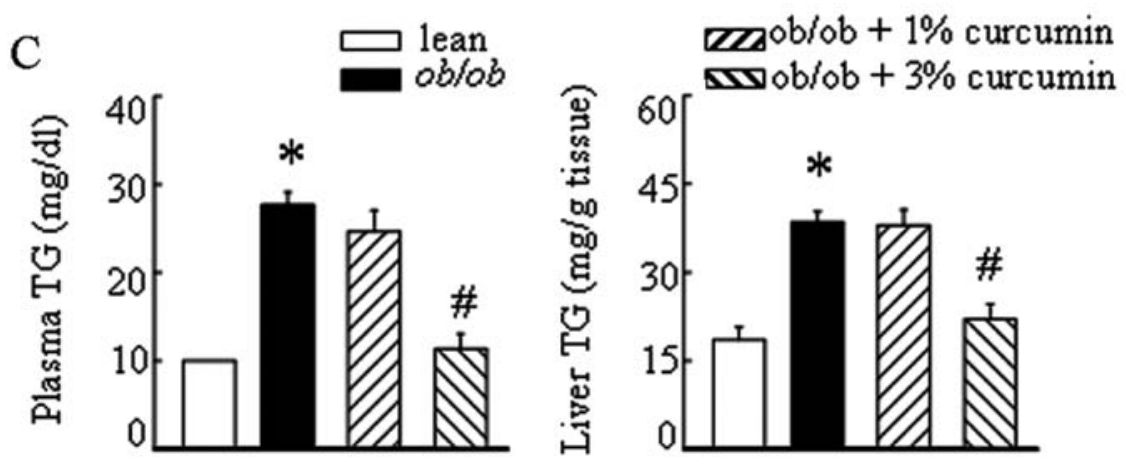

Figure 2. Curcumin inhibits hepatic steatosis and sterol regulatory element-binding protein-1c (SREBP-1c) expression in obese mice. (A) Histological analysis of liver sections from obese mice with or without curcumin treatment. Liver sections from obese mice fed with curcumin (3\% w/w) for 8 weeks. Upper panel, H\&E staining; lower panel, Oil red O staining. Original magnification, x40. (B) RT-PCR showing the effect of curcumin on obesity-induced SREBP-1c, acetyl-CoA carboxylase (ACC) and fatty acid synthase (FAS) gene expression. (C) Plasma triglyceride (TG) concentration (left panel) and hepatic triglyceride content (right panel). Data are presented as the means \pm SEM of 3 independent measurements. ${ }^{*} \mathrm{P}<0.05$ compared with lean control mice and ${ }^{\#} \mathrm{P}<0.05$ compared with obese mice without curcumin treatment $(n=5)$.

phosphorylation in the liver than lean control mice. Curcumintreated mice showed significantly different levels of F4/80 and STAT3 phosphorylation in the liver compared to obese mice (Fig. 3). In addition, suppressor of cytokine signaling 3 (SOCS3) protein levels were significantly decreased in obese mice treated with curcumin.

To verify that the attenuation of mitochondrial dysfunction signal by curcumin is a relevant molecular mechanism by which mitochondrial biogenesis is affected, obese mice were treated with curcumin. Obvious mtDNA copy numbers, boigenesis disorder and a decrease in mitochondrial respiratory chain (MRC) complex I activities and ATP levels were observed in the obese group compared to the lean control group (Fig. 4D). Since mitochondrial biogenesis was affected by curcumin treatment (Fig. 4E), we investigated whether curcumin affected the MRC. We found that complex I activity was altered after curcumin prevented the reduction of MRC in obese mice (Fig. 4D). We then determined whether obesity may affect both hepatic TBARS content and GSH levels. TBARS levels in obese mice were much higher compared to those of the lean control mice. Conversely, obesity led to a significant reduction in hepatic GSH levels. As expected, the results demonstrate that the presence of curcumin suppresses the TBARS generation and upregulates the GSH expression in obese mice. These observations suggest that curcumin attenuates obesity-induced aspects of mitochondrial dysfunction, including the depression of the GSH and the production of TBARS (Fig. 4B). 
A

A
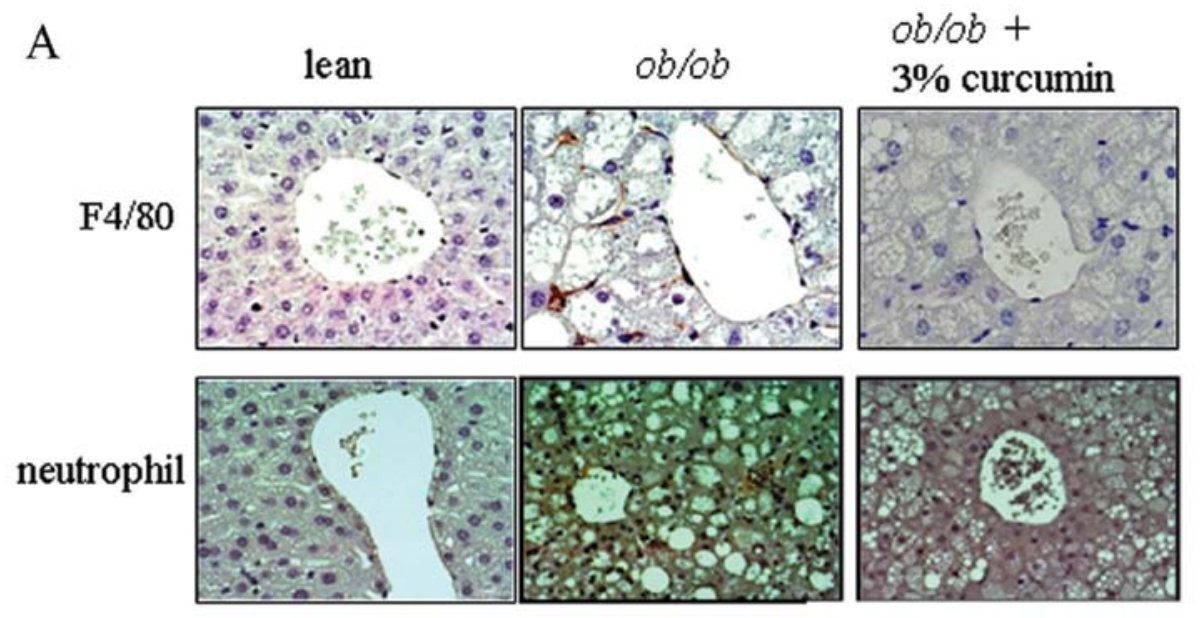

B
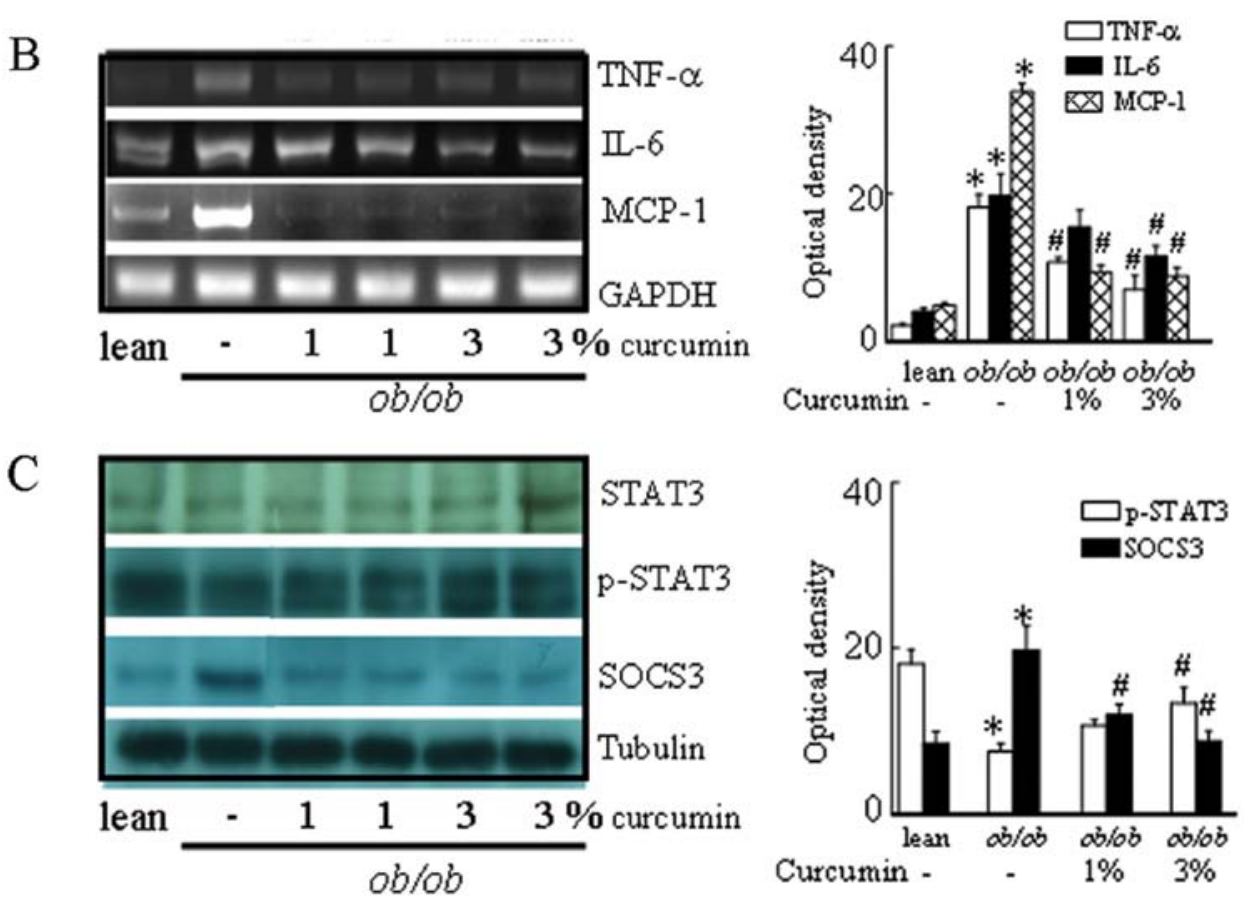

Figure 3. Effects of curcumin on hepatic macrophage recruitment and inflammatory response in obese mice. (A) Immunohistochemical staining of F4/80 (upper panel) and neutrophil (lower panel) in liver sections of obese mice. Original magnification, $\mathrm{x} 40$. (B) The hepatic tumor necrocis factor- $\alpha$ (TNF- $\alpha$ ), interleukin-6 (IL-6) and monocyte chemotactic protein-1 (MCP-1) mRNA levels were determined by RT-PCR and the results were normalized to GAPDH mRNA expression levels and regarded as an invariant control. (C) Western blot analysis representing the optical density of total signal transducer and activator of transcription 3 (STAT3) protein (upper panel) and its phosphorylated form (middle panel) and suppressor of cytokine signaling 3 (SOCS3) (lower panel) protein on liver homogenates. Data are presented as the means \pm SEM of 3 independent measurements. ${ }^{*} \mathrm{P}<0.05$ compared with lean control mice and ${ }^{\#} \mathrm{P}<0.05$ compared with obese mice without curcumin treatment $(\mathrm{n}=5)$.

\section{Discussion}

In the present study, we discovered a protective function of curcumin in hepatic steatosis. The protective role of curcumin may be observed in various animal models to different extents. Curcumin modulates multiple molecular targets and has potent anti-inflammatory activities, which may contribute to its therapeutic role in obesity and obesity-related metabolic diseases. To the best of our knowledge, our results demonstrate for the first time that curcumin-treated obese mice had decreased inflammatory response in AT, liver and blood. The decreases in inflammation with curcumin treatment may contribute to its beneficial effects on mitochondrial dysfunction associated with obesity-induced liver steatosis.
Weisberg et al (12) found that curcumin treatment of diabetic mice also decreased inflammatory response in AT, particularly in subcutaneous fat, as compared with diabetic controls. Based on the important role of inflammation in the development of obesity-linked metabolic abnormalities, the reduced inflammation in curcumin-treated obese mice may contribute to the improvement in hepatic steatosis observed in these mice. There are a number of observations in the literature linking adiposity with inflammation and increased liver disease. Several studies have conclusively demonstrated that a chronic low-grade systemic inflammatory state, predominantly arising from AT and liver, plays a critical role in the development of obesity-related diseases $(2,18,19)$. Thus, in AT, fat laden adipocytes initiate the inflammatory response by 

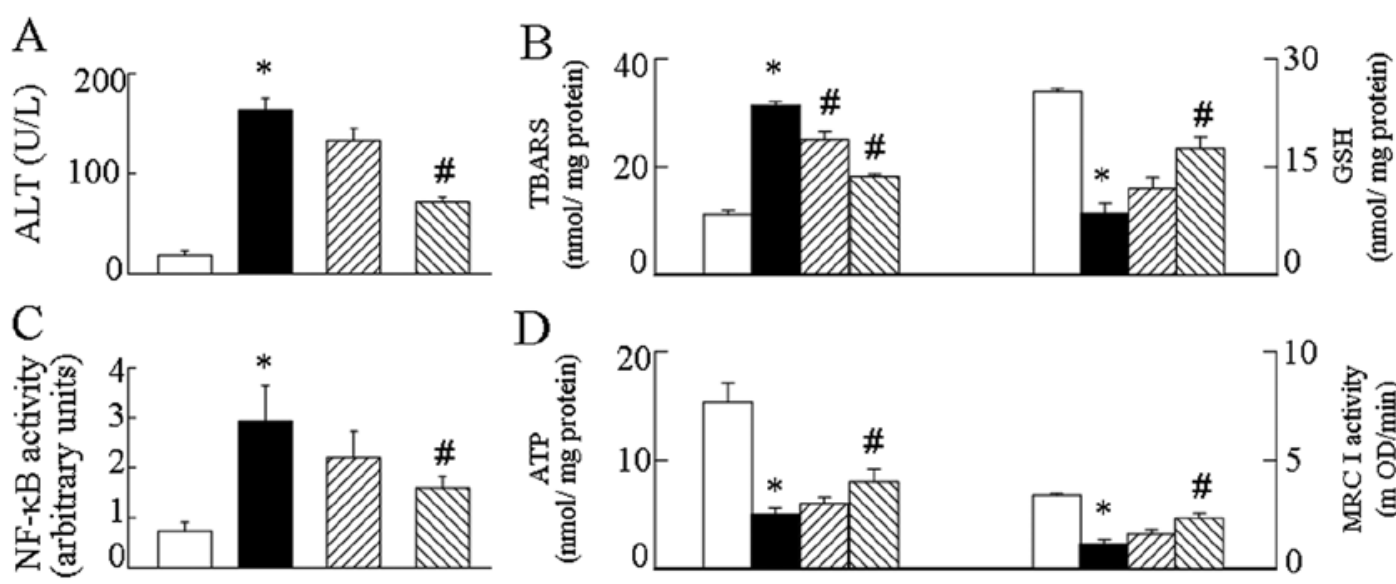

\section{D}
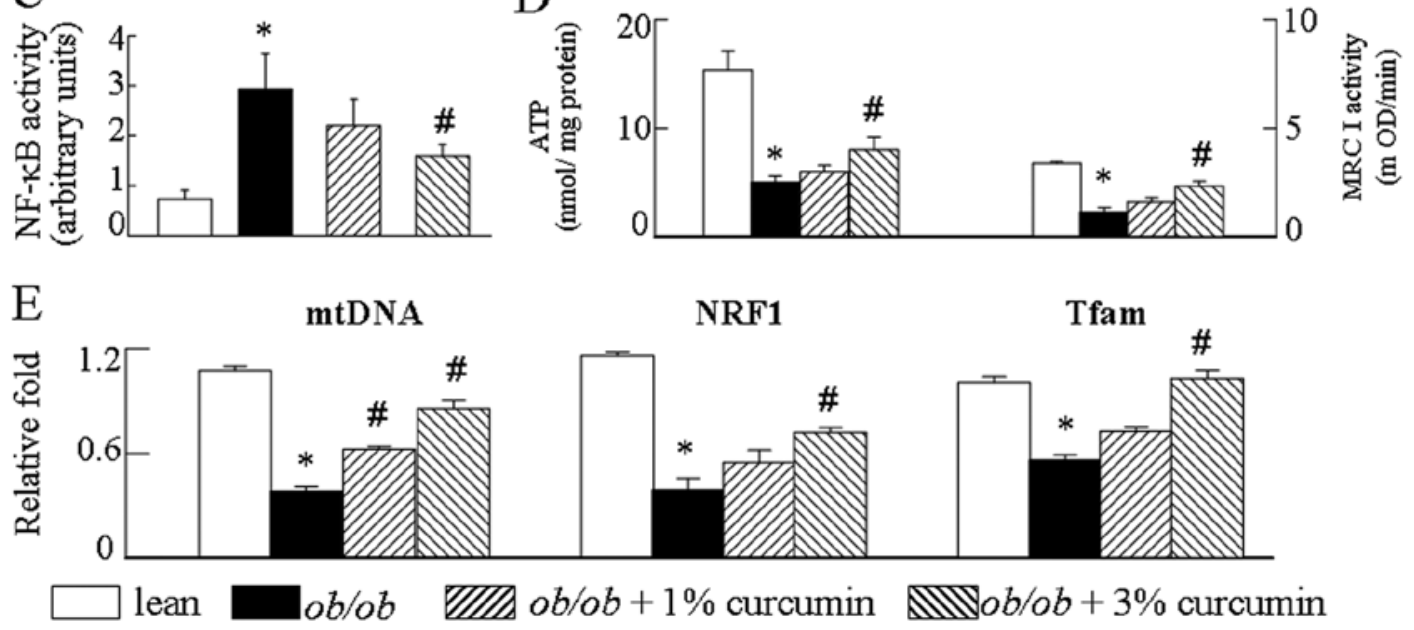

Figure 4. Curcumin attenuated oxidative stress and restored mitochondrial dysfunction in the liver of obese mice. Effects of curcumin on (A) alanine aminotransferase levels (ALT), (B) thiobarbituric acid reactive substances (TBARS) content and glutathione (GSH) levels, (C) nuclear factor- $\kappa \mathrm{B}$ (NF- $\kappa \mathrm{B})$ activity, (D) mitochondrial respiratory chain (MRC) activity and adenosine triphosphate (ATP) concentrations. Assays were performed as described in Materials and methods. (E) The mRNA level of mitochondrial DNA (mtDNA), nuclear respiratory factor 1 (NRF1) and transcription factor A (Tfam) were analyzed by real-time PCR. " $\mathrm{P}<0.05$ compared with lean control mice and ${ }^{\#} \mathrm{P}<0.05$ compared with obese mice without curcumin treatment ( $\mathrm{n}=5$ ).

producing cytokines and chemokines, including MCP-1 and TNF- $\alpha$, that promote the recruitment of pro-inflammatory macrophages and further amplification of local inflammation $(2,18,19)$. Increased AT inflammation contributes to insulin resistance by disrupting insulin-dependent signaling pathways, leading to the increased delivery of FFAs to the liver, and thereby contributing to hepatic steatosis (20). Moreover, fatty liver itself induces a local subacute inflammatory state characterized by the production of inflammatory mediators, such as TNF- $\alpha$ or IL-6, that directly contribute to hepatic and systemic insulin resistance (21).

The manifestations of the pro-inflammatory state include an increased production of TNF- $\alpha$ and MCP-1 and an upregulation of the NF- $\mathrm{NB}$ signaling pathway in the liver tissue. The inhibition of $\mathrm{NF}-\kappa \mathrm{B}$ signaling confers protection from obesity-induced inflammation in mouse models (22). Conversely, the stimulation of hepatic NF- $\mathrm{NB}$ signaling is sufficient to increase the local hepatic production of pro-inflammatory cytokines (23). The role of curcumin in obesity-associated inflammation $(12,24)$ and steatohepatitis (25) has been established. Therefore, the fact that curcumin attenuates the inflammatory response associated with obesity may prove useful in the medical management of patients with hepatic steatosis. On the other hand, the majority of studies have indicated that the STAT3-mediated signaling pathway is associated with hepatic steatosis. The activation of STAT3 in hepatocytes is known to ameliorate fatty liver. The overexpression of a constitutively active form of STAT3 in the liver of $\mathrm{db} / \mathrm{db}$ mice reduces the lipid content in the liver (26). The results from our present sudy show that treatment with curcumin induces STAT3 phosphorylation in the liver and alleviates steatosis in obese mice. We postulate that STAT3 activation is implicated in the protective function of curcumin in hepatic steatosis. In addition, the anti-steatogenic effect of curcumin is mediated, at least in part, via the inhibition of SREBP-1c, a master transcription factor that controls lipid synthesis, and subsequent suppression of hepatic lipogenesis. Our findings showed that the presence of decreased inhibitory signaling protein SOCS-3 expression and enhanced levels of its target, phosphorylated STAT3 in obese mice treated with curcumin. These significant findings shed light on a previous study by Ueki et al (27), who reported that the hepatic expression of SOCS-3 was elevated in the liver of obese mice and that the exogenous expression of the SOCS-3 protein induced SREBP-1c expression. Our findings show the concomitant downregulation of SOCS-3 with decreased SREBP-1c gene expression in the livers of obese mice treated with curcumin.

In addition to the effects on AT, obese mice also exhibit fatty liver and liver inflammation, which contributes substantially to obesity-linked mitochondrial dysfunction. A number of mechanisms may be considered to explain the mitochondrial dysfunction found in obese mice and NAFLD patients. One of them is oxidative stress. ROS-induced depletion in mtDNA may severely affect mitochondrial function and induces steatosis and liver lesion (28). Such depletion may impair the synthesis of complexes I, III, IV and V of the MRC. Our study demonstrates that TBARS, a marker of oxidative stress, were markedly increased in the liver of obese mice. Likewise, hepatic GSH was decreased in these animals. The 
decreased activity of the MRC complex I in obese mice is in part attributable to oxidative stress, as treatment of obese mice with curcumin, improved the activity of complex I of the MRC and inhibited the decline in ATP production by affecting energy metabolism in the mitochondria. Raised serum TNF- $\alpha$ levels have been demonstrated in patients with fatty liver or NASH. The role of this cytokine in the pathogenesis of mitochondrial dysfunction is strongly supported by the results of the current study, as the administration of curcumin to obese mice resulted in a marked increase in MRC activity. Based on the highly positive correlation of liver $N F-\kappa B$ activation with lipogenesis genes expression, the current results suggest that curcumin ameliorates fatty liver in obese mice and restores liver mitochondrial biogenesis. We demonstrate that the suppression of $\mathrm{NF}-\kappa \mathrm{B}$ enhances the ability of curcumin to rescue liver from obesity-induced mitochondrial dysfunction, strongly suggesting that a decrease in $\mathrm{NF}-\kappa \mathrm{B}$ activity by curcumin is at least partially responsible for this rescue of mitochondrial function.

Curcumin has been shown to prevent hypertriglyceridemia and hepatic NF- $\mathrm{NB}$ activation $(12,25,29)$. Our current study also indicates that curcumin activates STAT3 signaling and inhibits hepatic lipogenesis. We reveal that curcumin protects liver from obesity-induced steatosis and the possibility that the anti-inflammatory effect of curcumin is an integral part of the improvement of mitochondrial function in hepatic steatosis.

\section{Acknowledgements}

This study was supported by grant no. NSC98-2320-B-182021-MY3 (T.-Y.L.) from the National Science Council, Taiwan; and grant no. CMRPG5A0041 (H.-H.C.) from the Chang Gung Memorial Hospital, Linkuo, Taiwan.

\section{References}

1. Després JP and Lemieux I: Abdominal obesity and metabolic syndrome. Nature 444: 881-887, 2006.

2. Weisberg SP, McCann D, Desai M, Rosenbaum M, Leibel RL and Ferrante AW Jr: Obesity is associated with macrophage accumulation in adipose tissue. J Clin Invest 112: 1796-1808, 2003.

3. Wu H, Perrard XD, Wang Q, Perrard JL, Polsani VR, Jones PH, Smith CW and Ballantyne CM: CD11c expression in adipose tissue and blood and its role in diet-induced obesity. Arterioscler Thromb Vasc Biol 30: 186-192, 2010.

4. Sanyal AJ: AGA technical review on nonalcoholic fatty liver disease. Gastroenterology 123: 1705-1725, 2002.

5. Fabbrini E, Sullivan S and Klein S: Obesity and nonalcoholic fatty liver disease: biochemical, metabolic, and clinical implications. Hepatology 51: 679-689, 2010.

6. Tilg $\mathrm{H}$ and Moschen AR: Evolution of inflammation in nonalcoholic fatty liver disease: the multiple parallel hits hypothesis. Hepatology 52: 1836-1846, 2010.

7. Wang S, Kama t A, Pergola P, Swamy A, Tio F and Cusi K: Metabolic factors in the development of hepatic steatosis and altered mitochondrial gene expression in vivo. Metabolism 60: 1090-1099, 2011.

8. Chen J, Liu D, Bai Q, Song J, Guan J, Gao J, Liu B, Ma X and Du Y: Celecoxib attenuates liver steatosis and inflammation in non-alcoholic steatohepatitis induced by high-fat diet in rats Mol Med Rep 4: 811-816, 2011.
9. Hsieh PS, Lu KC, Chiang CF and Chen CH: Suppressive effect of COX2 inhibitor on the progression of adipose inflammation in high-fat-induced obese rats. Eur J Clin Invest 40: 164-171, 2010.

10. Shishodia S, Sethi G and Aggarwal BB: Curcumin: getting back to the roots. Ann NY Acad Sci 1056: 206-217, 2005.

11. Yekollu SK, Thomas R and O'Sullivan B: Targeting curcusomes to inflammatory dendritic cells inhibits NF- $\kappa \mathrm{B}$ and improves insulin resistance in obese mice. Diabetes 60: 2928-2938, 2011.

12. Weisberg SP, Leibel R and Tortoriello DV: Dietary curcumin significantly improves obesity-associated inflammation and diabetes in mouse models of diabesity. Endocrinology 149: 3549-3558, 2008

13. Koteish A and Mae Diehl A: Animal models of steatohepatitis. Best Pract Res Clin Gastroenterol 16: 679-690, 2002.

14. Lee TY, Chen FY, Chang HH and Lin HC: The effect of capillarisin on glycochenodexcholic acid-induced apoptosis and heme oxygenase-1 in rat primary hepatocytes. Mol Cell Biochem 325: 53-59, 2009.

15. Lee TY, Chang HH, Chen JH, Hsueh ML and Kuo JJ: Herb medicine Yin-Chen-Hao-Tang ameliorates hepatic fibrosis in bile duct ligation rats. J Ethnopharmacol 109: 318-324, 2007.

16. Tedesco L, Valerio A, Cervino C, Cardile A, Pagano C, Vettor R, Pasquali R, Carruba MO, Marsicano G, Lutz B, et al: Cannabinoid type 1 receptor blockade promotes mitochondrial biogenesis through endothelial nitric oxide synthase expression in white adipocytes. Diabetes 57: 2028-2036, 2008.

17. González R, Ferrín G, Hidalgo AB, Ranchal I, López-Cillero P, Santos-Gónzalez M, López-Lluch G, Briceño J, Gómez MA, Poyato A, et al: $\mathrm{N}$-acetylcysteine, coenzyme Q10 and superoxide dismutase mimetic prevent mitochondrial cell dysfunction and cell death induced by d-galactosamine in primary culture of human hepatocytes. Chem Biol Interact 181: 95-106, 2009.

18. Hotamisligil GS: Inflammation and metabolic disorders. Nature 444: 860-867, 2006.

19. Shoelson SE, Herrero L and Naaz A: Obesity, inflammation, and insulin resistance. Gastroenterology 132: 2169-2180, 2007.

20. Postic C and Girard J: Contribution of de novo fatty acid synthesis to hepatic steatosis and insulin resistance: lessons from genetically engineered mice. J Clin Invest 118: 829-838, 2008.

21. Parekh S and Anania FA: Abnormal lipid and glucose metabolism in obesity: implications for nonalcoholic fatty liver disease. Gastroenterology 132: 2191-2207, 2007.

22. Arkan MC, Hevener AL, Greten FR, Maeda S, Li ZW, Long JM, Wynshaw-Boris A, Poli G, Olefsky J and Karin M: IKK-beta links inflammation to obesity-induced insulin resistance. Nat Med 11: 191-198, 2005.

23. Arafa HM: Curcumin attenuates diet-induced hypercholesterolemia in rats. Med Sci Monit 11: 228-234, 2005.

24. Shehzad A, Ha T, Subhan F and Lee YS: New mechanisms and the anti-inflammatory role of curcumin in obesity and obesityrelated metabolic diseases. Eur J Nutr 50: 151-161, 2011.

25. Leclercq IA, Farrell GC, Sempoux C, dela Peña A and Horsmans Y: Curcumin inhibits NF-kappaB activation and reduces the severity of experimental steatohepatitis in mice. $\mathrm{J}$ Hepatol 41: 926-934, 2004.

26. Inoue $\mathrm{H}$, Ogawa W, Ozaki M, Haga S, Matsumoto M, Furukawa K, Hashimoto N, Kido Y, Mori T, Sakaue H, et al: Role of STAT-3 in regulation of hepatic gluconeogenic genes and carbohydrate metabolism in vivo. Nat Med 10: 168-174, 2004.

27. Ueki K, Kadowaki T and Kahn CR: Role of suppressors of cytokine signaling SOCS-1 and SOCS-3 in hepatic steatosis and the metabolic syndrome. Hepatol Res 33: 185-192, 2005.

28. Demeilliers C, Maisonneuve C, Grodet A, Mansouri A, Nguyen R, Tinel M, Lettéron P, Degott C, Feldmann G, Pessayre D and Fromenty B: Impaired adaptive resynthesis and prolonged depletion of hepatic mitochondrial DNA after repeated alcohol binges in mice. Gastroenterology 123: 1278-1290, 2002.

29. Li JM, Li YC, Kong LD and Hu QH: Curcumin inhibits hepatic protein-tyrosine phosphatase $1 \mathrm{~B}$ and prevents hypertriglyceridemia and hepatic steatosis in fructose-fed rats. Hepatology 51: $1555-1566,2010$. 